\title{
A two-dimensional Monte Carlo model for thin film growth by oblique evaporation: simulation of two-component systems for the example of $\mathrm{Co}-\mathrm{Cr}$
}

\author{
S. Müller-Pfeiffer ${ }^{1}$, H. van Kranenburg and J. C. Lodder \\ MESA Research Institute, University of Twente, Faculty of Electrical Engineering, P.O. Box 217, NL-7500 AE Enschede (Netherlands) \\ (Received September 24, 1991; accepted November 7, 1991)
}

\begin{abstract}
Starting from the well-known idea of atomic shadowing during thin film growth (i.e. ballistic aggregation) a two-dimensional computer model is developed which includes surface relaxation by thermally induced surface diffusion and atomic attraction for a two-component system. An attempt was made to link the model interaction energies to real bonding energies in $\mathrm{Co}-\mathrm{Cr}$ thin films. The model was applied to investigate structural as well as local configuration changes in the aggregates during systematic parameter variations.
\end{abstract}

\section{Introduction}

Thin films used in high performance optics, integrated circuit fabrication or magnetic storage technology are often deposited by physical vapour deposition techniques. These deposition processes are usually far from thermodynamic equilibrium. The resulting structural and physical properties of the films are essentially influenced by the deposition conditions. For the development of films with desired properties it is useful to get an insight into the mechanisms of structure formation in thin films, even if the growth models are still very crude and the influence of the film structure on its physical properties is known only qualitatively in many cases.

One material of both scientific and technological interest is $\mathrm{Co}-\mathrm{Cr}$, which is used as a medium for high-density magnetic recording. The magnetic properties of $\mathrm{Co}-\mathrm{Cr}$ films are strongly influenced by the deposition conditions during film growth. For example, the saturation magnetization $M_{\mathrm{s}}$ and coercive field $H_{\mathrm{c}}$ depend on the degree of inhomogeneity of the distribution of both sorts of atoms in the films (segregation), which in turn is influenced by, for example, the deposition temperature [1].

With the present model we do not explain the structure development or even the magnetic properties of $\mathrm{Co}-\mathrm{Cr}$ films comprehensively, but intend to obtain a

\footnotetext{
'On leave from the Institute of Technical Physics, Helmholtzweg 4, D(O)-6900 Jena, FRG
}

more qualitative insight into the development of the morphology of these films.

\section{Physical basis of the applied model}

In the following report we describe a two-dimensional hard-disc Monte Carlo model, which will be used to study the changes in both morphology and local arrangement in thin films manufactured by evaporation. The simulated films will consist of a maximum of 50000 particles, thereby restricting the film thickness to approximately $600 \AA$. The films consist of two different species of atoms, which to some degree will be identified in Section 2.3 with $\mathrm{Co}$ and $\mathrm{Cr}$ atoms in $\mathrm{Co}-\mathrm{Cr}$ thin films. The evaporation situations to be covered include the oblique evaporation of both species at different incidence angles from one source as well as the evaporation of each species from a separate source.

\subsection{Experimental data and basic considerations}

Thin films manufactured by one of the various physical vapour deposition methods have a microstructure, which in general differs from the structure of bulk material of the same average composition. This microstructure depends on the conditions during the film formation and is governed by the kinetics of the growth process. In the case of oblique deposition the deposition geometry also plays an essential role in the formation of the microstructure. Understanding the morphology will also help us to understand in more detail the origin of the physical properties of thin films. Despite the great 
complexity of structure formation in thin films, their morphological properties can be systematized for a large number of materials in terms of a small number of reduced deposition parameters.

Such a phenomenological scheme based on systematization of experimental data was first given by Movchan and Demchishin [2] as the structure zone model, where the morphology (i.e. presence, shape and size of columns, crystallites etc.) of the thin film was related to the reduced substrate temperature $T / T_{\mathrm{M}}(T$, substrate temperature; $T_{\mathrm{M}}$, melting point of the material) during deposition. Based on this model and our experiments we choose a $T / T_{\mathrm{M}}$ value below 0.3 for the simulations.

The following structure growth philosophy for thin films can be derived from the experimental data: the morphology of the film is a result of a number of competing processes, finally resulting in aggregation and relaxation. Aggregation only would lead to very sparse structures in the films, while various relaxation processes tend to drive the film towards thermodynamic equilibrium. These processes interact in such a complex way that the estimation of the result is only possible by computer simulation.

\subsection{Description of the model, its parameters and their connection to the deposition process}

The starting point for developing the growth model is the ballistic aggregation process of hard spheres, introduced by Leamy et al. [3] for the explanation of the columnar structure in evaporated thin films. However, the approach of sticking atoms was too crude and for a better explanation of the experimental results the ballistic aggregation model was further developed, first by Henderson et al. [4] by introducing an appropriate relaxation scheme. Models similar to that of the authors but using a simpler diffusion mechanism were investigated by Bartholomeusz [5] and Smith [6].

The model presented here is two dimensional and the particles are represented as hard discs. Consequently, the film grows in the $z$ direction and will be compared with a $y-z$ section of a growing film. In this connection the use of some usual terms, well known from experiments, such as "column", "bulk" and "internal surface" need additional explanation. Columns are coherent regions in the aggregate, more or less parallel to each other and separated by voids, void regions or trenches. Bulk atoms in our simulation are atoms which have six next neighbours, i.e. atoms which are completely surrounded by other atoms. Atoms at internal surfaces are atoms with less than six next neighbours, which are not situated at the surface.

As we consider an evaporation process, the particles move on straight parallel trajectories towards the film surface (straight because the mean free path is large in comparison with typical dimensions of thin film structures) until they collide with an already deposited atom or with a substrate atom. The angle of incidence, which is the angle of the incoming vapour flux $x_{i}$, and all other angles are measured from the film normal. After the collision, and taking into account the usually noncentral impact of the incoming atom on the captured atom, it relaxes into the next stable position (cradle) right or left of the capture atom. During this process all the kinetic energy of the incoming atom will be dissipated.

Immediately before reaching the film surface, the trajectory of the particle in real evaporation does not remain straight because of the finite interatomic potential (e.g. van der Waals forces) between the approaching atom and the surface atoms. The deviation from the straight trajectory, which will be a few ångströms, depends on the particle's kinetic energy and the interaction energy between the surface atoms and the incoming particle. In order to include, to a certain extent, the influence of this effect in the model and regarding the kinetic energy of the incoming particles on the growing structure, the hard sphere sticking of the incoming atom onto the deposit will be replaced by hard sphere sticking with a capture radius greater than the particle radius. In the following we give the justification of this approach and also give an estimation of the capture radius.

For the estimation of the enhanced capture radius we use a simple continuum model for the surface evolution of thin films. This means that we derive a differential equation for the evolution of the film's surface $h(y, t)$, where $h(y, t)$ should be a single-valued function. Consequently, the internal structure of the films cannot be considered, i.e. the film is assumed to be amorphous. The description of voids and overhangs is also excluded in this case. We consider three contributions to the evolution equation for $h(y, t)$ : the contribution caused by the atomic shadowing effect $v_{\text {shad }}$, the contribution resulting from the atomic attraction $v_{\text {attr }}$ and that arising from the capillarity-driven surface diffusion $v_{\text {sid }}$. Consequently we obtain

$\frac{\partial}{\partial t} h(y, t)=v_{\text {shad }}+v_{\text {attr }}+v_{\text {sdf }}$

for a system with atomic attraction. The contribution arising from shadowing can be written according to Sahni et al. [7] as

$v_{\text {shad }}=J r_{\mathrm{p}} \Delta h_{y y}\left(1+\Delta h_{y}^{2}\right)^{-1 / 2}$

where the following notation was used: $J$ is the deposition rate, $r_{\mathrm{p}}$ is the particle radius, $\Delta h=h(y, t)-J t$ and $\partial(\Delta h) / \partial y=\Delta h_{y}$, etc. 
According to Shevchik [8] the atomic attraction term in the evolution eqn. (1) has the following form

$$
v_{\mathrm{attr}}=\frac{J L^{2}}{4 E_{0}} \frac{\partial^{2}}{\partial x^{2}} \int_{-x}^{+\infty} \bar{U}\left(y-y^{\prime}\right) \Delta h\left(y^{\prime}, t\right) \mathrm{d} y^{\prime}
$$

where

$$
\bar{U}\left(y-y^{\prime}\right)=\frac{1}{L} \int_{0}^{L} U\left(y-y^{\prime}, z^{\prime}\right) \mathrm{d} z^{\prime}
$$

We used the following notation: $U$, interaction potential; $L$, potential range of $U$; and $E_{0}$, kinetic energy of the incoming particles. The contribution of the capillarity-driven surface diffusion according to Mullins [9] has the form

$v_{\mathrm{sfd}}=-D_{\mathrm{e}} \frac{\partial}{\partial x}\left\{\left(1+\Delta h_{y}^{2}\right)^{-1 / 2} \frac{\partial}{\partial x}\left[\Delta h_{y y}\left(1+\Delta h_{y}^{2}\right)^{-3 / 2}\right]\right\}$

where

$D_{\mathrm{e}}=D_{\mathrm{s}} \sigma_{\mathrm{s}} \Omega^{2} / k_{\mathrm{B}} T$

In eqn. (6) we used: $D_{s}$, surface diffusivity; $\sigma_{s}$, isotropic surface energy density; $\Omega$, atomic volume; and $k_{\mathrm{B}} T$, thermal energy.

Now, the stability of eqn. (1) can be investigated, which means that we will investigate under which conditions a little sinusoidal perturbation

$\delta h(y, t=0)=\alpha \sin (k y)$

where $\alpha \ll 1$, will decay or grow. For this purpose we perform a Fourier transformation of eqn. (1) with regard to eqns. (2), (3) and (5). If we neglect higher orders in $k$, it yields

$$
\begin{aligned}
\frac{\partial}{\partial t} \hat{h}(k, t)= & -\frac{J L^{2}}{4 E_{0}} k^{2} \hat{U} \hat{h}(k, t)+J r_{\mathrm{p}} k^{2} \hat{h}(k, t) \\
& -D_{\mathrm{e}} k^{4} \hat{h}(k, t)
\end{aligned}
$$

Equation (7) can be rewritten as

$$
\hat{h}(k, t)=\hat{h}(k, 0) \exp \left\{\left[r_{\mathrm{p}} J k^{2}+\frac{J L^{2}}{4 E_{0}} k^{2} \hat{U}(k)-D_{\mathrm{e}} k^{4}\right] t\right\}
$$

and further as

$$
\hat{h}(k, t)=\hat{h}(k, 0) \exp \left[\left(r_{\mathrm{p}}^{\prime} J k^{2}-D_{\mathrm{e}} k^{4}\right) t\right]
$$

where we introduced

$r_{\mathrm{p}}^{\prime}=r_{\mathrm{p}}-\frac{L^{2}}{4 E_{0}} \hat{U}(k)$

From eqn. (9) we can derive the stability condition for eqn. (1). If stability should be fullfilled, then

$r_{\mathrm{p}}^{\prime} J k^{2}-D_{\mathrm{e}} k^{4}<0$
Equation (10) can be reinterpreted in such a way that $r_{p}^{\prime}$ is the capture radius in the case where attractive forces exist, so we can write $r_{c}$ instead of $r_{p}^{\prime}$. If we approximate the interaction potential by an exponential potential

$U(r)=-\frac{A}{a^{2}} \exp \left(\frac{-r}{L}\right)$

where $a$ is the interatomic spacing, $A$ the potential parameter and $L$ the potential range, then if $k L \ll 1$ we obtain

$\frac{r_{\mathrm{c}}}{r_{\mathrm{p}}}=1+\frac{A L^{3}}{4 E_{0} a^{2} r_{\mathrm{p}}}$

For a reasonable set of parameters $\left(A=1 \mathrm{eV}, L=3 r_{\mathrm{p}}\right.$, $\left.a=2 r_{\mathrm{p}}, E_{0}=A-10 A\right) r_{\mathrm{c}} / r_{\mathrm{p}}$ will be in the range $1.17<$ $r_{\mathrm{c}} / r_{\mathrm{p}}<2.68$. In the simulations we use $r_{\mathrm{c}} / r_{\mathrm{p}}=1.2$.

After reaching the surface and the first relaxation to a relatively stable cradle position the surface atom will not usually reside there. The deposit will relax due to surface diffusion. We limit our model principally to relatively low substrate temperatures $\left(T / T_{\mathrm{M}}<0.5\right)$ because above $T / T_{\mathrm{M}}=0.5$ the volume diffusion will be important, which is impossible to model with such a hard sphere packing model. According to the structure zone model originally developed by Movchan and Demchishin [2] in this temperature region the main relaxation process will be surface diffusion. This process will be modelled by thermally induced hopping and is assumed to follow the Boltzmann statistics.

In the present model this process will be implemented as a Monte Carlo procedure. The bonding energy of an atom in the deposit or at its surface is assumed to be isotropic (spherical atoms), additive and depends only on the two atoms involved in the bond. No other atoms influence the strength of this particular bond. The latter condition is important, because in general the value of the interaction energy between atom $A$ and atom $B$ depends on the electronic state of both atoms, which again may depend on the other neighbours of both atoms. Then the bonding energy $E_{j}$ of an individual atom $j$ can be calculated easily as

$E_{j}=\sum_{j \in n(j)} \Phi_{s(i) s(j)}$

In eqn. (12) $s(i)$ denotes the sort of particle $i, \Phi_{\alpha \beta}$ the energy of one bond of an atom of sort $\alpha$ and an atom of sort $\beta$ and $n(j)$ denotes the set of the next neighbours of atom $j$. Next neighbours will be considered as discs which are at a distance $a$, where $a$ is in the range $2 r_{\mathrm{p}} \leqslant a \leqslant \xi r_{\mathrm{p}}$. In our simulation $\xi=5^{1 / 2}$ taking into account the box potential between the particles. The choice of the width and depth of the box potential is in general arbitrary but, to guarantee that an atom has always less than seven next neighbours, the constraint 
$\xi<\sin (\pi / 7)$ must be fulfilled, which is achieved by making $\xi=5^{1 / 2}$. It is assumed that the energy of an individual bond is only dependent on the atoms taking part in it. If the atom $j$ is a surface atom, the energy $E_{j}$ is equal to the sublimation energy barrier of this particular atom.

With this approach the diffusion barrier can be calculated as well, if we assume that the diffusion barrier energy is equal to the sum of the energies of the bonds to be broken for a particular jump. In general, for a particular atom, it will depend on the jumping direction since the sort and the number of bonds to be broken can differ for jumps in different directions:

$Q_{j, \text { direction }}=\sum_{\text {bonds to be broken }} \Phi_{s(i) s(j)}$

For assumed Boltzmann statistics we obtain the following jumping rates $R_{\alpha}$ for different atoms and configurations denoted as $\alpha$ :

$R_{x}=v \exp \left(-Q_{\alpha} / k_{\mathrm{B}} T\right)$

where $Q_{x}$ is the diffusion barrier depending on the situation (i.e. number of next neighbours, their species, jump direction), $v$ is the phonon frequency in the deposit (typically about $10^{13} \mathrm{~Hz}$ ), $k_{\mathrm{B}}$ is Boltzmann's constant and $T$ is the substrate temperature. From the diffusion rates $R_{x}$ the maximum rate $R$ equals the diffusion rate of the most mobile surface atoms. This rate can be compared with the deposition rate $J$ (in terms of monolayers per second).

Quite naturally a dimensionless parameter $d_{1}=R / J$ occurs, which gives the mean number of diffusional jumps for the most mobile atoms during the time needed for the deposition of one monolayer. This parameter $d_{1}$ incorporates both substrate temperature and deposition rate into the model. Strictly speaking, the correct use of $d_{1}$ is restricted to the most mobile surface atoms, because changing the temperature also influences the ratio of the jumping rates for atoms with different bonding energies. However, the latter effect is assumed to be less important, because the main relaxation effect will be caused by the most mobile atoms, and we assume that the morphology is less dependent on the other less mobile surface atoms.

For metals (one component and in three dimensions) there is a simple empirical formula by Neumann and Hirschwald [10] for the dependence of the diffusion barrier on the temperature and on the melting point of the material:

$Q=\left(5+\frac{20 T}{3 T_{\mathrm{M}}}\right) k_{\mathrm{B}} T_{\mathrm{M}}$

This formula can be used to obtain an approximate idea about the model parameter $d_{1}$ for different temperatures and deposition rates.
TABLE 1. Temperature dependence of the diffusion parameter $d_{1}$ in a one-component system

\begin{tabular}{lc}
\hline$T / T_{M}$ & $d_{1}$ \\
\hline 0.15 & $4 \times 10^{-4}$ \\
0.20 & 2 \\
0.25 & 260 \\
0.30 & 7350 \\
\hline
\end{tabular}

If we collect the previous results from eqns. (14) and (15) and the definition of $d_{1}$, we obtain the following:

$d_{1}=J^{-1} v \exp \left(-20 / 3-5 T_{\mathrm{M}} / T\right)$

In order to have an idea about the parameters, $d_{1}$ is listed for several temperatures in Table 1, where $v$ is assumed to be $10^{13} \mathrm{~Hz}$ and $J$ to be 0.1 monolayer $\mathrm{s}^{-1}$.

The transition in the Movchan-Demchishin model from zone I to zone II appears at $T / T_{\mathrm{M}} \approx 0.3$ and is caused by the onset of surface diffusion above $T / T_{\mathrm{M}}=0.3$. However, in our two-dimensional model the onset of diffusion coincides, according to eqn. (16), with the transition temperature given by $T / T_{\mathrm{M} \text {.trans }}=$ $0.19\left(d_{1}=0.5\right)$. Consequently, we cannot scale the transition temperature from the three-dimensional experiment to our two-dimensional model on a one-to-one basis.

At the start of the simulation program a monolayer of a priori immobile atoms is generated which, after the generation is finished, serves as the substrate. For the simulation we chose an amorphous substrate as described in ref. 11 .

2.3. Bonding energies for cobalt, chromium and $\mathrm{Co}-\mathrm{Cr}$ In order to compare our simulation results with experiments we identify the particle sorts denoted as $\alpha$, $\beta$ and so on with real atoms. The main task will be to find out relevant bonding energies for these atoms. An interesting system for this purpose is the $\mathrm{Co}-\mathrm{Cr}$ thin film system because of its technical relevance and the great amount of information available for it. We shall now refer to some results that are important for the justification of our approach and for the illustration of effects to be expected.

Haines [12] reported about theoretical considerations concerning the dependence of the saturation magnetization $M_{\mathrm{s}}$ on the composition in $\mathrm{Co}-\mathrm{Cr}$ thin films deposited at different temperatures. There it was stated that the saturation magnetization for a composition without $\mathrm{Cr}-\mathrm{Cr}$ bonds will be less than in a random mixture of $\mathrm{Co}$ and $\mathrm{Cr}$ atoms. The state with fewer or no $\mathrm{Cr}-\mathrm{Cr}$ bonds is energetically favourable and the temperature-driven surface diffusion during growth tends to push the system into that state. An increased temperature favours this effect, and we should observe a 
transition from the state of random mixture to the non-random arrangement with increasing substrate temperature. On the other hand it is known (e.g. see ref. 1) that an increasing substrate temperature in a certain temperature range during deposition leads to segregation in the $\mathrm{Co}-\mathrm{Cr}$ films.

Band structure calculations on $\mathrm{Co}-\mathrm{Cr}$ alloys by $\mathrm{Nel}$ son et al. [13] yielded an average charge transfer of approximately 0.04 electrons from chromium to cobalt for each existing $\mathrm{Co}-\mathrm{Cr}$ bond. Since this charge will fill the $3 \mathrm{~d}$ shell of cobalt, this leads to a decrease of the magnetic moment of the affected Co atom. This charge transfer and charge distribution are not sensitive to stress and to the percentage of chromium present in the alloy. Summarizing the calculated data it can be stated that for the electronic properties of $\mathrm{Co}-\mathrm{Cr}$ alloys the local arrangement of cobalt and chromium in the unit cell is unimportant and the interaction energy of an atom in the $\mathrm{Co}-\mathrm{Cr}$ matrix can be treated as the sum of the bonding energies as proposed in the present model.

In order to obtain information about the $\mathrm{Cr}-\mathrm{Cr}$ and Co-Co bonding energies we consider both the sublimation enthalpy and the dissociation energy listed in reference books such as ref. 14. From both values it can be stated that the $\mathrm{Co}-\mathrm{Co}$ bond is approximately 1.07 times stronger than the $\mathrm{Cr}-\mathrm{Cr}$ bond. Information about the strength of $\mathrm{Co}-\mathrm{Cr}$ bonds is not so easy to obtain; it should be stated here that the thermodynamic data on the alloying enthalpy is not very useful, because besides the changes of the internal energy there are also the changes in entropy due to configurational transitions. This contribution cannot be easily estimated.

Therefore we use data from Miedema's semiempirical theory $[15,16]$ about the interfacial energy of solidliquid metal combinations. These considerations yield for the dissolving of both chromium in liquid cobalt and cobalt in liquid chromium a negative dissolving enthalpy (positive interaction energy) of about $-18 \mathrm{~kJ}$ per mole of solvent. Comparing these data with the above-mentioned data about the $\mathrm{Cr}-\mathrm{Cr}$ and $\mathrm{Co}-\mathrm{Co}$ bonds, we can state that the $\mathrm{Co}-\mathrm{Cr}$ bonding energy is about 1.12 times stronger than the $\mathrm{Cr}-\mathrm{Cr}$ bond. Therefore we shall use the following bonding energies in the model in arbitrary energy units (e.u.):

$$
\begin{aligned}
& \Phi_{\mathrm{CrCr}}=1.00 \text { e.u. } \\
& \Phi_{\mathrm{CoCo}}=1.07 \text { e.u. } \\
& \Phi_{\mathrm{CoCr}}=1.12 \text { e.u. }
\end{aligned}
$$

\section{Modelling results}

With the model introduced above and its parameters we carried out the following experiments: evaporation of the $\mathrm{Co}-\mathrm{Cr}$ mixture from one source at four different angles between $0^{\circ}$ and $45^{\circ}\left(\phi_{0}=0^{\circ}, 15^{\circ}, 27.5^{\circ}, 45^{\circ}\right)$ and at five different surface diffusion parameters $\left(d_{1}=0,75\right.$, $290,1170,2400)$ for each incidence angle. All the deposition experiments were done for approximately 10000 particles. Additionally, for comparison the series for an incidence angle of $45^{\circ}$ was repeated with the deposition of 50000 particles to study the system size dependence of the results. Another experiment was the deposition of the material from two sources symmetrically positioned, so that according to the conventions chromium was evaporated under a deposition angle of $-27.5^{\circ}$ and cobalt under an angle of $27.5^{\circ}$.

\subsection{Implementation and verification of the model}

The model program was written in Pascal and is available in an MS-DOS and an Apollo version. The MS-DOS version was used for the relatively small simulations while the larger ones were performed on an Apollo workstation. The simulation is time consuming; the deposition of 10000 particles at a diffusion parameter $d_{1}=2400$ takes more than $30 \mathrm{~h}$ on a $16 \mathrm{MHz} 386 \mathrm{SX}$ personal computer.

Before systematically varying the model parameters it was verified that neither the limited system size nor the use of the random number generator provided by the operating system of the computer resulted in artefacts. The independence of the results of the system size was checked by comparing simulations with 10000 and simulations with 50000 particles, obtained from one parameter set under the common angle of incidence $\phi_{0}=45^{\circ}$. Neither series showed significant differences in column inclination or in column diameter. One example of such a comparison can be seen from Figs. 1 and 2(d). The verification of the random number generator was performed by running simulations with identical parameter sets but different random number generators. The resulting structures did not show striking differences.

\subsection{Morphology of the $\mathrm{Co}-\mathrm{Cr}$ mixture obtained from one source}

In Figs. 2(a) -2(d) we see a simulated microstructure for an angle of incidence of $\phi_{0}=45^{\circ}$. Figures $2(\mathrm{a})-2(\mathrm{~d})$ show the structure with increasing diffusion. Figure 3 does the same, but for a different angle of incidence, namely $15^{\circ}$. As can be seen from both Figs. 2 and 3 we obtain in the whole parameter field coherent aggregates of material, which will be called columns.

We found for the whole series that the inclination angle of the columns increases with increasing incidence angle for every fixed surface diffusion; compare Fig. 2(a) with Fig. 3(a) and Fig. 2(b) with Fig. 3(b) etc. Further, we found a decreasing column inclination with increasing surface diffusion. Although methods have 


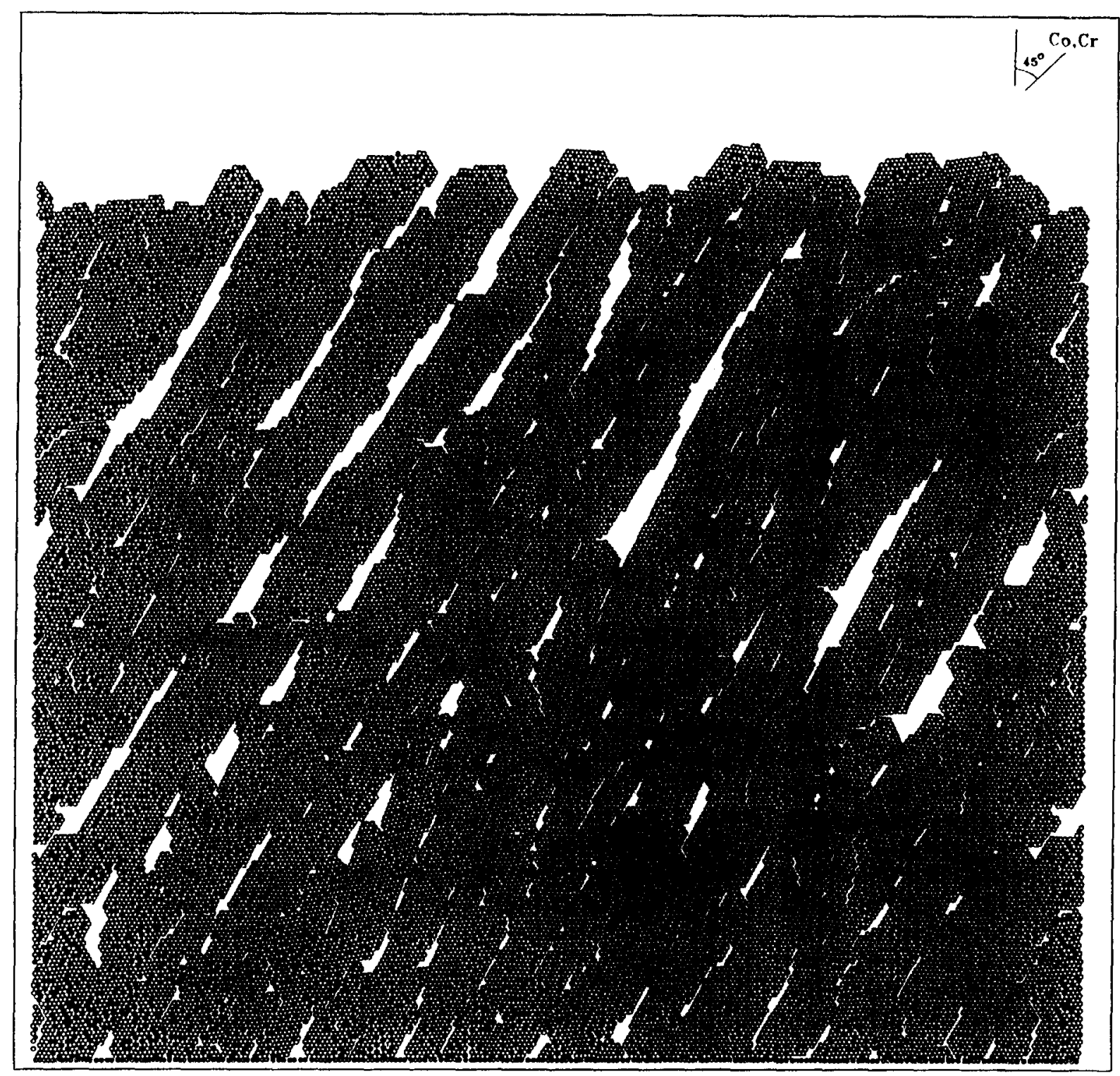

Fig. 1. Result of the simulation run for $\mathrm{Co}-\mathrm{Cr}$ with $22 \mathrm{at} \% \mathrm{Cr}$ (black) with the incidence angle $\phi_{0}=45$ for surface mobility $d_{1}=2400$ $\left(N=50000\right.$ particles. $r_{\mathrm{c}} / r_{\mathrm{p}}=1.25$, interaction energies as listed in the text).

been described for the determination of column inclination and spacing directly from the data in the literature (see ref. 17 and 18) we will not use them, because in many of our cases they did not yield better results than visual interpretation of the images. The last results are given in Table 2. The columnar diameter increased with increasing surface diffusion, as can be seen in Figs. 2 and 3 .

We did not observe any significant growth of the column diameter with growing film thickness, which was to be expected with the simulated film thickness. This observation was also confirmed by the large experiments with the 50000 particles.

We also studied the morphology of the deposit by a very simple method - determining the distribution of atoms with different numbers of next neighbours, as can be seen in Table 3. From the results we can conclude that the share of bulk atoms, i.e. atoms with six next neighbours, only depends on the surface diffusion and not on the angle of incidence. The data in Table 3 are too inaccurate to conclude any significant changes in the distribution of the atoms due to a change in the incidence angle. We can tell from the simulated structures that with an increasing angle of incidence the void size increases, causing the film density to decrease. However, the number of internal surfaces in the film is almost constant, as can be seen from Table 3 .

In comparison with real thin films grown under similar mobility conditions the column diameter in our experiment is much too small. In reality $\mathrm{Co}-\mathrm{Cr}$ columns (under similar deposition conditions) have a 

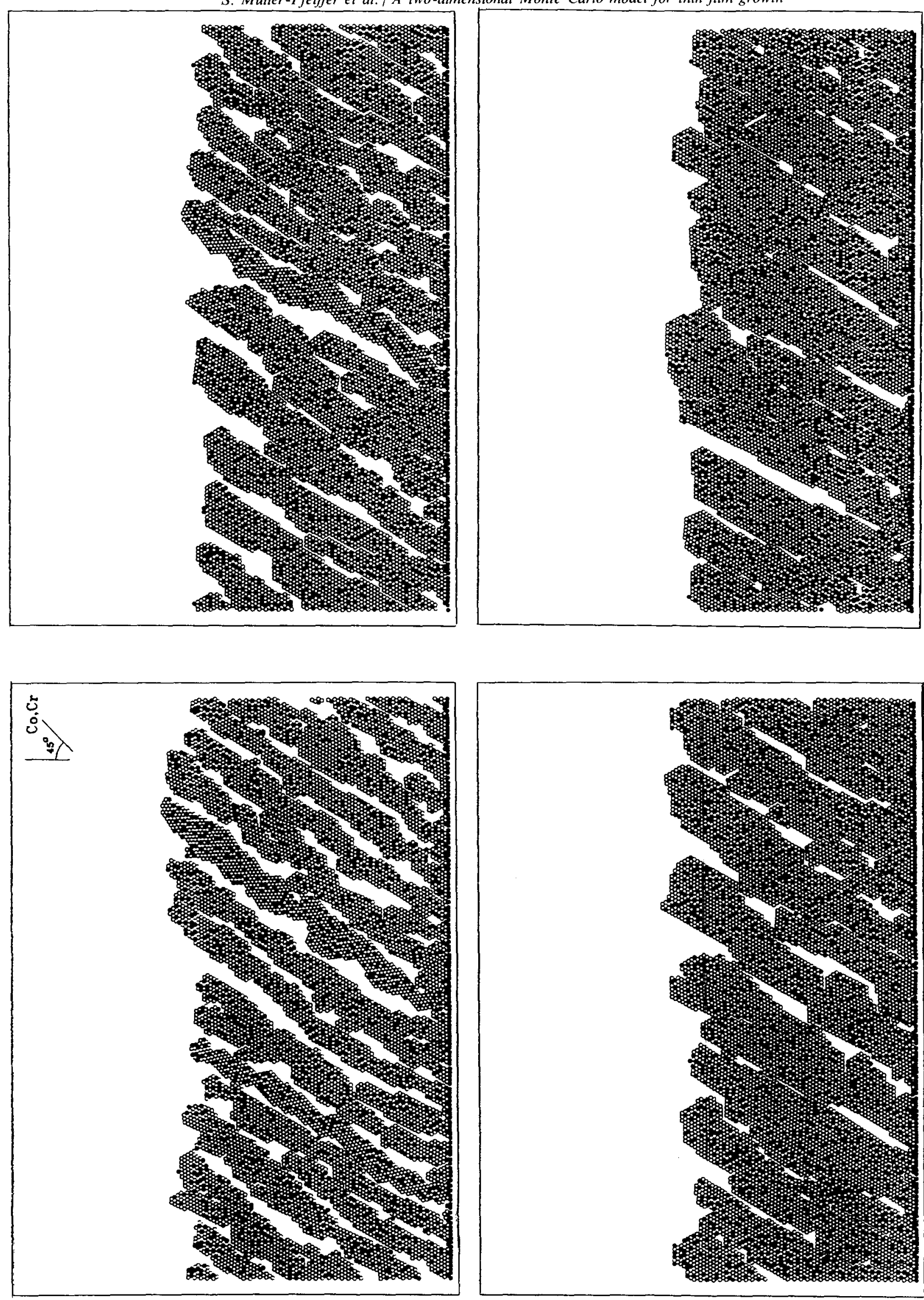

㟧

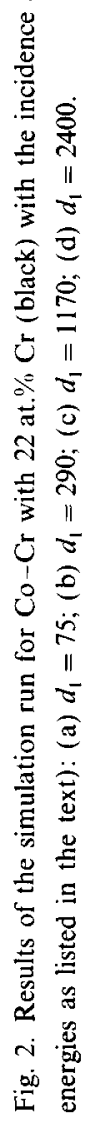




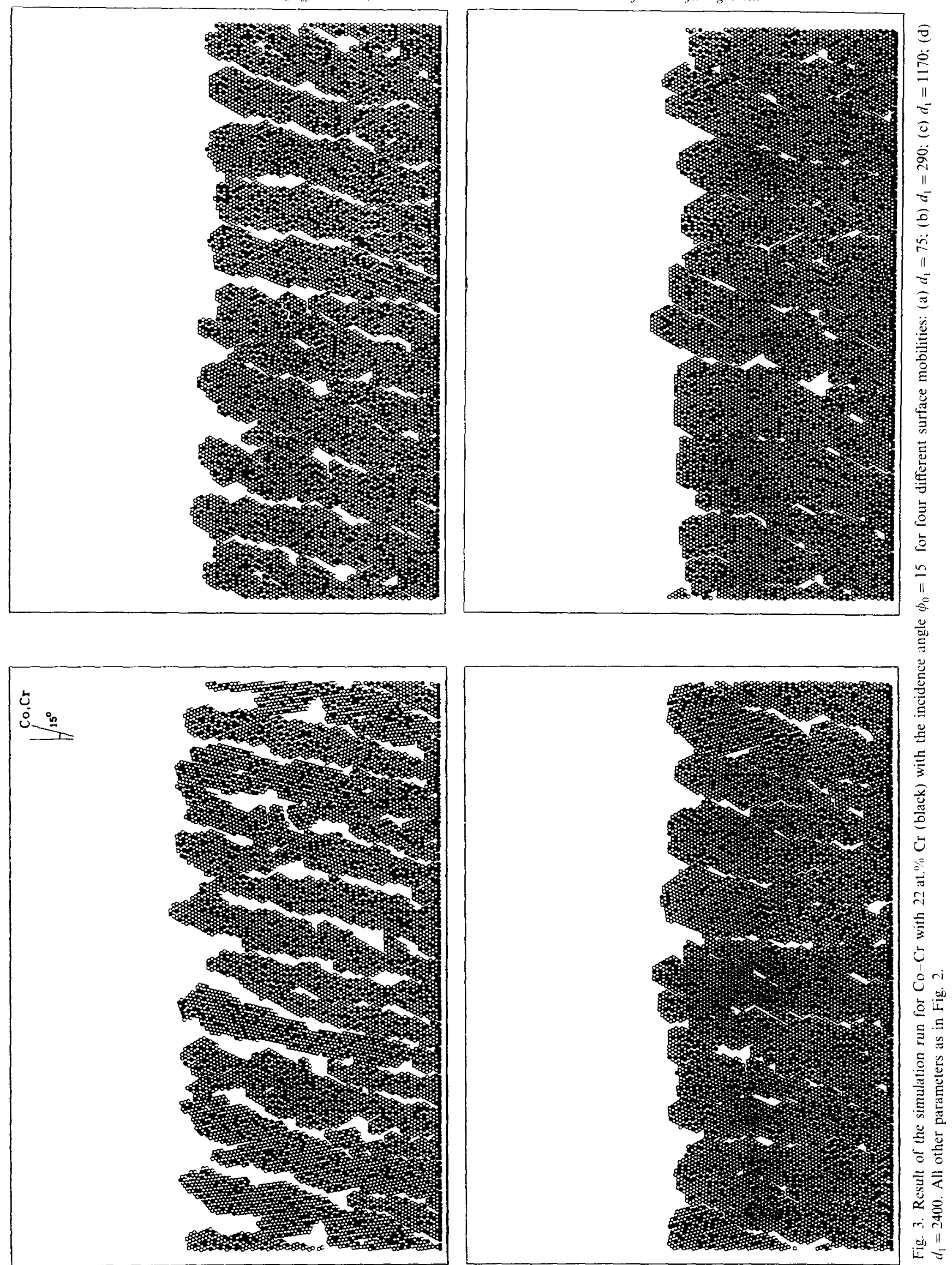


TABLE 2. Average column inclination (measured from the film normal) at $22 \% \mathrm{Cr}$

\begin{tabular}{|c|c|c|c|c|c|}
\hline \multirow[t]{2}{*}{$d_{1}$} & \multicolumn{4}{|c|}{ One-source deposition } & \multirow{2}{*}{$\begin{array}{l}\text { Two-source deposition } \\
\text { results }{ }^{\mathbf{b}}\end{array}$} \\
\hline & $0^{\text {oit }}$ & $15^{\circ}$ & $27.5^{\circ}$ & $45^{\circ}$ & \\
\hline 0 & - & - & - & - & - \\
\hline 75 & 0 & 14 & 24 & 35 & 12 \\
\hline 290 & 0 & 14 & 23 & 33 & 10 \\
\hline 1170 & 0 & 11 & 22 & 34 & 8 \\
\hline 2400 & 0 & 10 & 22 & 27 & 7 \\
\hline
\end{tabular}

TABLE 3. Distribution of the number of next neighbours $N$ for three different diffusion parameters

\begin{tabular}{rrrrrrr}
\hline \multicolumn{1}{l}{$d_{1}$} & \multicolumn{1}{c}{$\phi_{0}$} & $N=2$ & $N=3$ & $N=4$ & $N=5$ & $N=6$ \\
\hline 0 & 0.0 & 3.77 & 15.36 & 29.81 & 28.71 & 22.36 \\
& 15.0 & 4.24 & 14.76 & 29.43 & 29.31 & 22.25 \\
& 27.5 & 3.67 & 14.70 & 29.74 & 27.33 & 24.53 \\
& 45.0 & 3.87 & 14.35 & 29.72 & 26.29 & 25.78 \\
292 & 0.0 & 0.16 & 5.07 & 11.99 & 11.52 & 71.26 \\
& 15.0 & 0.17 & 4.92 & 12.64 & 10.19 & 72.26 \\
& 27.5 & 0.09 & 4.79 & 14.32 & 13.09 & 67.67 \\
& 45.0 & 0.09 & 4.94 & 15.45 & 9.17 & 70.35 \\
2370 & 0.0 & 0.07 & 3.44 & 9.81 & 9.75 & 76.95 \\
& 15.0 & 0.04 & 3.80 & 9.96 & 9.45 & 76.75 \\
& 27.5 & 0.07 & 3.32 & 10.97 & 11.17 & 74.84 \\
& 45.0 & 0.05 & 2.67 & 11.98 & 9.38 & 75.91 \\
\hline
\end{tabular}

diameter of some $10-50 \mathrm{~nm}$, i.e some $40-200$ atomic diameters. However, in our computer experiments we obtained column diameters of some 10 atoms. In our opinion this has two main reasons as follows.

Especially in the high temperature region, i.e. under conditions with a considerable surface diffusion, in the two-dimensional experiment (with a one-dimensional substrate) we get an average nucleus distance which is much too low in comparison with three-dimensional data. In addition there is a difference in diffusion direction possibilities for the two- and three-dimensional cases.

It is to be expected that in a system with "soft" interaction instead of a hard sphere packing, and which allows collective motion of the atoms, a lot of voids and chains of voids would collapse resulting in larger columns and voids. In this case columns would be separated by nanocracks.

Nevertheless the simulation provides an insight into the morphology, which is very helpful for the interpretation of the deposition process.

\subsection{Non-random local arrangement of atoms in modelled $\mathrm{Co}-\mathrm{Cr}$}

It is mentioned in the literature (e.g. see ref. 1) that for

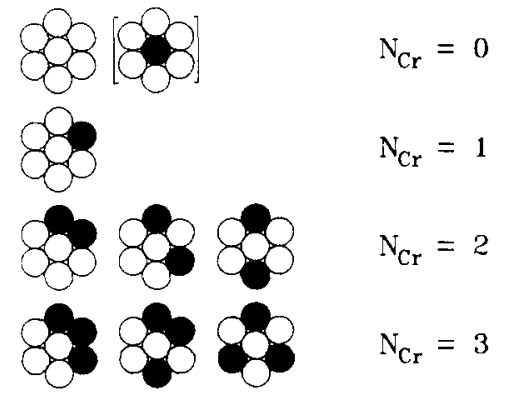

Fig. 4. Different possibilities of local arrangement of next chromium neighbours around Co atoms.

$\mathrm{Co}-\mathrm{Cr}$ thin films a chromium segregation is important for the magnetic properties of these films. Segregation means in this context that the $\mathrm{Cr}$ atoms will not be distributed homogeneously in the $\mathrm{Co}-\mathrm{Cr}$ matrix. This effect will be investigated in the modelled films by means of quantification of the next-neighbour arrangements.

In order to obtain more detailed information on the local distribution we determine local next-neighbour distribution functions in the following way: $P_{\alpha, \beta}(N, K)$ will denote the percentage of $\alpha$ atoms with $N$ next neighbours which have among these $N$ next neighbours $K \beta$ atoms normalized to all $\alpha$ atoms with $N$ next neighbours ( $\alpha$ and $\beta$ denote the atom species such as cobalt or chromium). It should be mentioned that for $K=2,3,4$ we do not distinguish between the different cases of local arrangement, as shown in Fig. 4. The experimentally determined distributions $P_{x, \beta}$ will be compared with the similar distributions of a random $\alpha-\beta$ mixture.

For a random mixture of two different species of atoms $\alpha$ and $\beta$ (i.e. cobalt and chromium) where the species $\beta$ (i.e. chromium) is contained with a concentration of $C(0 \leqslant C \leqslant 1)$, we obtain for the probability $P_{(\alpha, \beta) . \alpha}(N, K)$ that among the $N$ next neighbours of a given $\alpha$ atom are $K \beta$ atoms ( $\mathrm{Cr}$ atoms):

$P_{(x, \beta), \beta}^{\mathrm{rand}}(N, K)=\frac{N !}{(N-K) ! K !} C^{K}(1-C)^{N-K}$

The distributions $P_{\mathrm{Cr}, \mathrm{Cr}}(N, K)$ and $P_{\mathrm{Co}, \mathrm{Cr}}(N, K)$ were experimentally determined for $N=6,5,4$ in all simulations.

First, by comparing all the experimental $P_{\alpha, \beta}$, we note a significant difference between the $d_{1}=0$ experiments and all others. The $d_{1}=0$ experiment yielded the same distributions as expected for the random mixture. All other experiments yielded other distributions. These distributions do not show any significant behaviour during the systematic variation of both the angle of incidence and the surface diffusion.

That is the reason why all the distributions $P_{\mathrm{Cr}, \mathrm{Cr}}(N, K)$ and $P_{\mathrm{Co}, \mathrm{Cr}}(N, K)$ were averaged over all $d_{1} \neq 0$ and all angles of incidence for each $N=6,5,4$. 


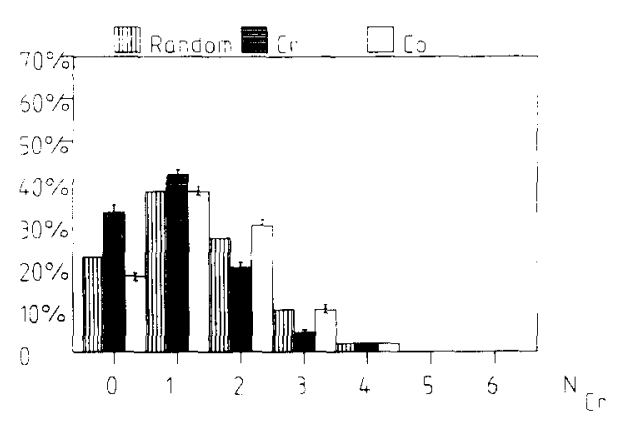

Fig. 5. Local distribution of $\mathrm{Cr}$ atoms around $\mathrm{Co}$ atoms and $\mathrm{Cr}$ atoms with six next neighbours for $d_{1}>0$ in comparison with the distribution in a random mixture: $\square$, cobalt; 11 , chromium; $I T$, random.

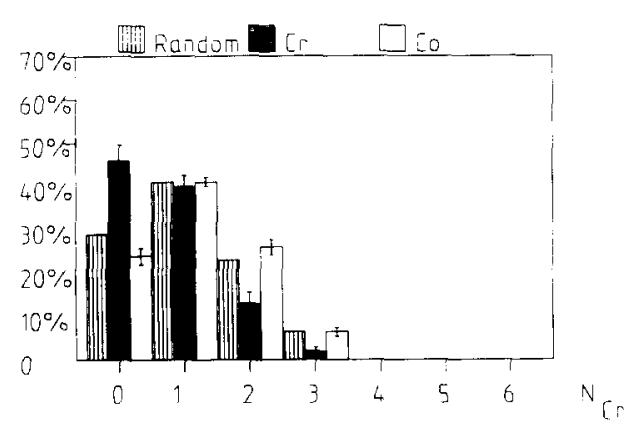

Fig. 6. Local distribution of $\mathrm{Cr}$ atoms around $\mathrm{Co}$ atoms and $\mathrm{Cr}$ atoms with five next neighbours for $d_{1}>0$ in comparison with the distribution in a random mixture; $\square$, cobalt; + I, chromium; ....., random

These averaged $P_{\mathrm{Cr}, \mathrm{Cr}}^{\mathrm{avg}}(N, K)$ and $P_{\mathrm{Co} . \mathrm{Cr}}^{\mathrm{avg}}(N, K)$ were compared with $P_{(x, \beta), C r}^{r a n d}(N, K)$ from eqn. (17) for $C_{\mathrm{Cr}}=0.22$ and are shown in Figs. 5-7. The open bars in all figures show the distributions $P_{\mathrm{Co}, \mathrm{Cr}}^{\mathrm{avg}}(N, K)$ around the Co atoms, the full bars the distribution $P_{\mathrm{Cr}, \mathrm{Cr}}(N, K)$ around the $\mathrm{Cr}$ atoms and the bars with vertical shading the expected distribution for the random mixture. As an example, the first bar with horizontal shading in Fig. 5 shows the average share of $\mathrm{Cr}$ atoms without chromium next neighbours normalized to all $\mathrm{Cr}$ atoms with six next neighbours obtained from the averaged experimental data.

As can be seen from Figs. 5-7 we obtain differences from the random mixture. The number of Co atoms with only cobalt (i.e. without chromium next neighbours) is less than in a random mixture for all $N$. The number of Co atoms with two chromium next neighbours is larger than in a random distribution for all $N$. The number of Co atoms with $K(K \neq 0,2)$ next chromium neighbours is not different from the random distribution. The number of $\mathrm{Cr}$ atoms without chromium next neighbours is larger than for the random mixture, while the number of $\mathrm{Cr}$ atoms with more than zero chromium next neighbours is less than for the random mixture. This behaviour is not surprising, because it is generated by the bonding energies put into the model.

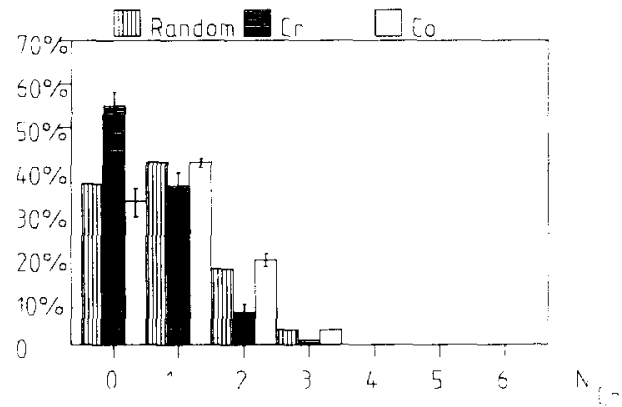

Fig. 7. Local distribution of $\mathrm{Cr}$ atoms around $\mathrm{Co}$ atoms and $\mathrm{Cr}$ atoms with four next neighbours for $d_{1}>0$ in comparison with the distribution in a random mixture: $\square$, cobalt; 1 , chromium; $\bar{L}$, random.

All these results are significant, as can be seen from the size of the error bars in Figs. 5-7. For one incidence angle $\left(\phi_{0}=27.5^{\circ}\right)$ these local distributions were also determined for lower values of $d_{1}$ $\left(d_{1}=37,18,9,4,0.4\right)$. These cases yielded the same local distributions as shown in Figs. 5-7. Hence also at very low surface diffusions the local arrangement of the atoms was strongly influenced.

\subsection{Two-source evaporation}

With the above-described method we also studied the results on both the morphology and the arrangement of $\mathrm{Co}$ and $\mathrm{Cr}$ atoms in thin films deposited from two sources, as described by two of the authors in ref. 19. The experimental set-up is sketched in Fig. 8.

From the series of experiments - one of them is shown in Fig. 8 - we found that the average angle of inclination of the columns in the coevaporation case is less than in the one-source evaporation (see also Table 2). This behaviour can be explained by an average flux of the incoming atoms at an angle that is less than $27.5^{\circ}$. Further, we observed a strong process-induced segregation, i.e. an increased amount of chromium at the side of the columns towards the chromium source and large areas with almost pure cobalt at the cobalt side of the columns.

Finally, with increasing $d_{1}$, i.e. with increasing diffusion, the chromium is distributed more homogeneously in the columns. In agreement with the experimental films [19] this shows a transition from the process-induced segregation to the thermally enhanced segregation.

\section{Concluding remarks}

Despite its relative simplicity we believe that the model contains the main physical processes governing thin film growth during evaporation. It fits into the general concept that thin film morphology and also local order are a result of competing aggregation and relaxation processes, as predicted by the structure zone model. In the case 


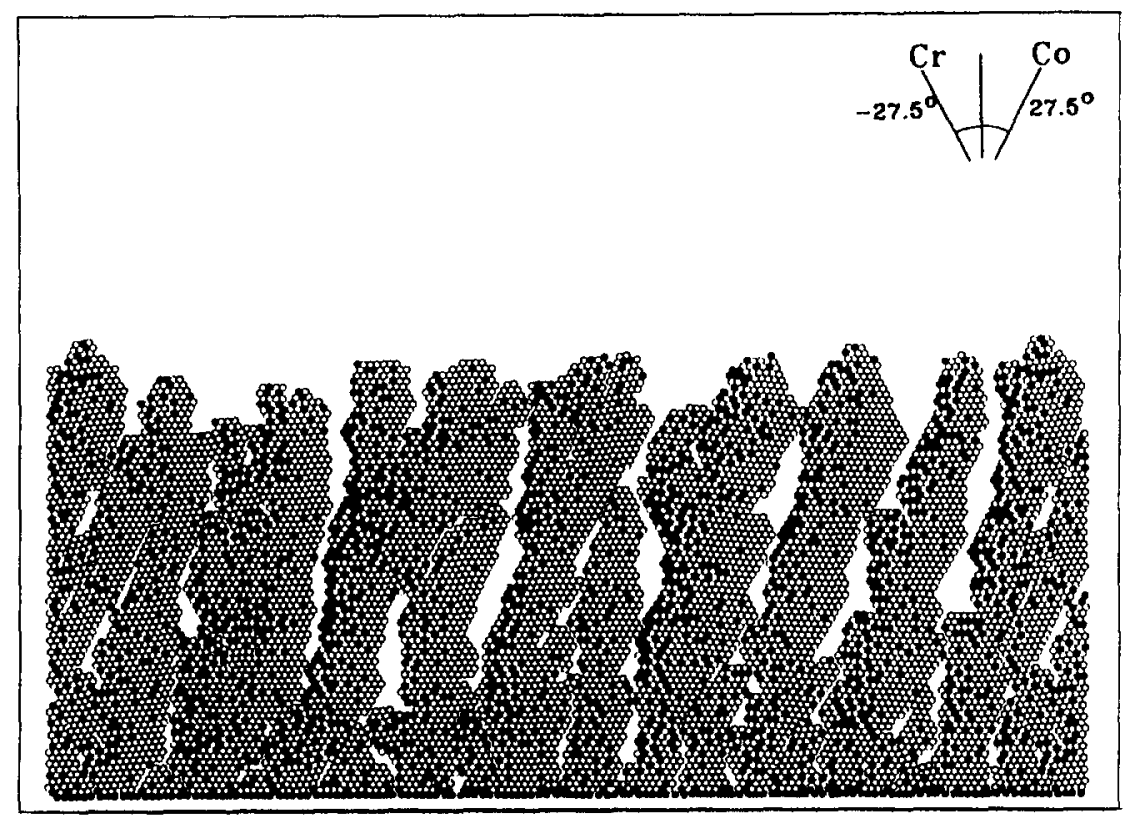

Fig. 8. Result of a simulation run for $\mathrm{Co}-22$ at. $\% \mathrm{Cr}$ from two-source evaporation with the following parameters: $\phi_{\mathrm{Cr}}=-27.5^{\circ}, \phi_{\mathrm{Co}}=$ $27.5^{\circ}$ and $d_{1}=290$. All other parameters as in Fig. 2.

of evaporation studied here they are represented as ballistic aggregation and surface diffusion respectively.

Quantities such as column diameter and column inclination are difficult to compare with analogous parameters from the model. This is mainly caused by the two-dimensionality of the model (the problem of energetic parameter choice in two dimensions, too high nucleation density on a one-dimensional substrate and the absence of different crystallographic packings) and the use of hard spheres instead of particles with a more realistic interaction (no void collapse, no internal stresses).

Despite all its difficulties, the model shows some of the right tendencies during parameter variation such as an increase of the column diameter with increasing surface diffusion and offers the possibility of comprehensive characterization (statistical measurements) of the resulting structure.

Further work should be directed towards solving the above-mentioned problems, i.e. it should include a three-dimensional model, and towards inclusion of physical processes typical of sputtering.

\section{Acknowledgments}

The authors are grateful to the Community Action on Magnetic Storage Technology of the European Community which financially supported the stay of one of them (S. Müller-Pfeiffer) at the University of Twente.

\section{References}

1 Y. Maeda and M. Asahi, IEEE Trans. Magn., 23 (1987) 2061.

2 B. A. Movchan and A. V. Demchishin, Phys. Met. Metallogr., 28 (1969) 83.

3 H. J. Leamy, G. H. Gilmer and A. G. Dirks, Curr. Top. Mater. Sci., 6 (1980) 309

4 D. J. Henderson, M. H. Brodsky and P. Chaudhary, Appl. Phys. Lett., 25 (1974) 641

5 B. Bartolomeusz, Proc. Soc. Photo-Opt. Instrum. Eng., 821 (1987) 174

6 D. J. Smith, Proc. Soc. Photo-Opt. Instrum. Eng., 821 (1987) 120.

7 P. S. Sahni, D. J. Srolovitz, G. S. Grest and B. G. Bukiet, Phys. Rev. Lett., 60 (1988) 424

8 N. Shevchik, J. Non-Cryst. Solids, 12 (1973) 141.

9 W. W. Mullins, J. Appl. Phys., 28 (1957) 333.

10 G. Neumann and W. Hirschwald, Z. Phys. Chem., Abt. B,8I (1972) 163

11 S. Müller-Pfeiffer, H.-J. Anklam and W. Haubenreißer, Phys. Status. Solidi B, 160 (1990) 491.

12 G. W. Haines, J. Appl. Phys., 55 (1984) 2263.

13 J. S. Nelson, C. Y. Fong and J. P. Batra, J. Appl. Phys., 63 (1988) 3049.

14 CRC Handbook of Chemistry and Physics, CRC Press, Boca Raton, FL, 1983.

15 A. R. Miedema, Z. Metallkde., 69 (1978) 455.

16 A. R. Miedema and F. J. A. den Broeder, Z. Metallkde., 70(1979) 14.

17 B. J. Bartholomeusz, K.-H. Müller and M. R. Jacobson, Proc. Soc. Photo-Opt. Instrum. Eng., 120 (1987) 2.

18 M. Sikkins, I. J. Hodgkinson, F. Horowitz, H. A. MacLeod and J. J. Whator, Opt. Eng., 25 (1986) 142.

19 H. van Kranenburg, J. C. Lodder, Y. Maeda, L. Toth and T. j. A. Popma, IEEE Trans. Magn., 26 (5) (1990) 1620. 